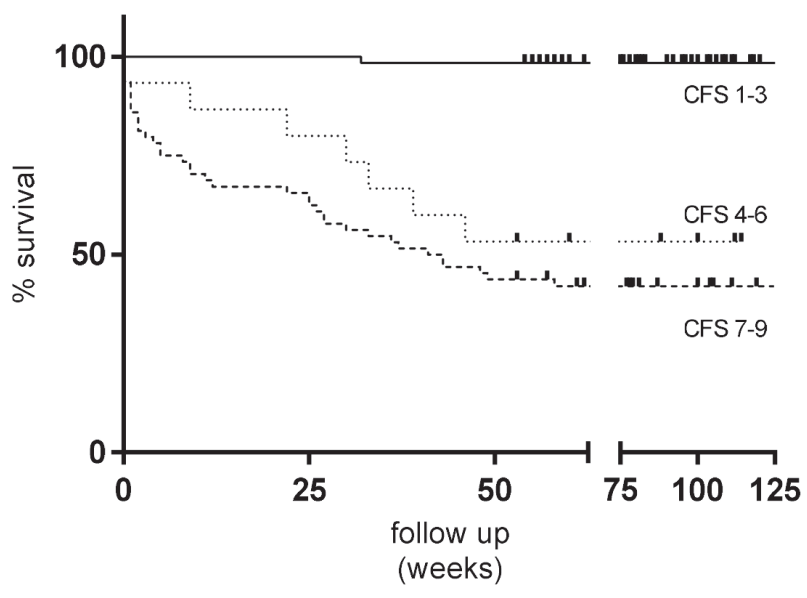

Abstract PTH-42 Figure 1 Kaplan-Meier curves of survival, grouped according to the CFS

Although Blatchford scores $>2$ were not a predictor of early outcome or the use of intervention, there is a correlation between Blatchford score and death over time. The Blatchford score is significantly greater in those with survival $<4$ weeks (14.6) compared with those $<52$ weeks (8.9, $\mathrm{p}<0.01)$. Likewise pre and post Rockall score is correlated with 52 week survival, ( $\log$ rank test, $\mathrm{p}<0.01$ ), but a poor indicator of the need to intervene at OGD, or death from GI causes. Indicating that both risk assessment scores are in fact surrogate markers of frailty rather than GI pathology.

All patients that died during admission had a CFS 7-9, and of the $15(11 \%)$ patients that died $<4$ weeks $80 \%$ had CFS 7-9. Of the 23 patients who died $<12$ weeks $91 \%$ had a CFS 7-9. Only $1 / 64(1.5 \%)$ of patients with a CFS $1-3$ survived $<52$ weeks.

Conclusion A Blatchford score $\leq 2$ is strongly predictive of no AUGIB.

No scoring system predicts reliably whether intervention was required. A high percentage of those patients with poor outcomes during admission, and $<12$ weeks have a CFS of 7 9 , in contrast to the excellent long term survival of those patients with a CFS 1-3.

The Rockwood CFS can help differentiate those patients who may have poor outcomes from undertaking emergency OGD, particularly in the short term, giving an opportunity to discuss and pursue a more conservative approach.

\section{PTH-43 IMPROVING ENDOSCOPY TURNAROUND TIME AND LIST EFFICIENCY THROUGH IMPLEMENTATION OF A MULTI- FACETED IMPROVEMENT PLAN}

${ }^{1}$ Karishma Sethi Arora*, ${ }^{2}$ Chehkuan Tai, 'Laura Marelli. 'Homerton University Hospital, London, UK; ${ }^{2}$ The Royal Free Hospital, London, UK

\subsection{6/gutjnl-2021-BSG.358}

Introduction Maximising efficiency in endoscopy in the face of increasing service pressure, demand and complexity of endoscopy is imperative, particularly given the unparalleled impact of the COVID pandemic on service delivery and cancer pathways. Previous attempts to improve turnaround time through introduction of a turnaround nurse have been hindered by inadequate staffing. We aimed to improve list efficiency and improve turnaround time through the application of marginal gains theory and implementation of a multi-faceted improvement plan.

Methods Data was collected from electronic patient records and audit entries made by endoscopy staff. List 'actual' start and stop times were audited against 'scheduled' times. Turnaround time was assessed by a Quality Improvement (QI) Fellow, observing the endoscopy list and collecting information in real time. Results were discussed at a departmental meeting and a 4-stage improvement plan was devised and implemented. Re-audit data was collected to determine effect.

Results Preliminary audit data revealed $89 \%$ of list starts to be delayed. Lists ran beyond scheduled stop times in $73 \%$. The improvement plan saw:

1) Review and re-organisation of the nursing rota.

2) Departmental commissioning of an external 'change agent' to conduct interviews with nursing and endoscopy managers and work with the QI fellow in designing a bespoke team-building day to address communication strategies and brainstorm perceived departmental issues hindering efficiency.

3) Introduction of an in-room debrief tool, to enhance situation awareness and teamwork.

4) Implementation of a multi-modal 'Nurse-led consent' training programme, combining didactic and in-situ scenariobased simulation training, measuring and recording competence using Directly-Observed-Procedural-Skills (DOPS) assessments.

Re-audit data revealed improved list finish-times (49\% cf. $27 \%$ ) although delays in start-times remained. Median turnaround time was 10 minutes, a major improvement from a turnaround time in 20.8 minutes in 2018.

Conclusions It is recognised that single improvement interventions are unlikely to result in significant, sustainable change. The aggregation of marginal gains theory dictates that small, marginal gains can add up to a remarkable improvement. Our 4-stage improvement plan saw the implementation of a revised nursing rota and a bespoke team-building day in tandem with the introduction of a 'Nurse Consent' training programme and a novel team debrief tool. In this way, we were able to implement change, whilst simultaneously assessing and addressing staff morale, engage key stakeholders and as a result significantly improve turnaround time. We plan to streamline admission and patient preparation processes to further address delayed start times in future cycles of the improvement project.

\section{PTH-44 IMPROVING THE MORECAMBE BAY ACUTE UPPER GASTROINTESTINAL BLEEDING SERVICE}

Christopher Mysko*, Rami Mohamed, Ahmed Hamdy, Firas Elfourtia, John Keating. University Hospitals Morecambe Bay NHS Foundation Trust, UK

\subsection{6/gutjnl-2021-BSG.359}

Introduction Acute upper gastrointestinal bleeding (AUGIB) is a common medical emergency with an estimated incidence of 134 per 100,000 population; 1 presentation every 6 minutes. Mortality is high at approximately $10 \%$ and is largely unchanged over the last two decades with UK-wide audits revealing variability and poor care standards despite the development of guidelines. The British Society of Gastroenterology (BSG) recently published the 'AUGIB Care Bundle' to address deficiencies and drive sustained improvements. ${ }^{1}$ 